\title{
ANALOG VERY LARGE SCALE INTEGRATION IMPLEMENTATION OF DENDRITE SEGMENT WITH VOLTAGE DEPENDENT SPIKING BEHAVIOR
}

\author{
K. K. O'Hanlon, R. B. Grove, R. P. Ramachandran, and L. M. Head \\ Department of Electrical and Computer Engineering, Rowan University, Glassboro, NJ, USA
}

\begin{abstract}
This paper describes a set of aVLSI circuits and a subsystem composed of these circuits that reproduce the electrical functionality of a dendrite.

Keywords - Neuromorphic Engineering, Dendrite, Neuron
\end{abstract}

\section{INTRODUCTION}

The first silicon retina, designed by Mead and Mahowald in the late 1980's [1], was an analog Very Large Scale Integration (aVLSI) system. The success of early Integrated Circuit (IC) implementations of biologically accurate neural circuits led to the exploration of many other computational systems, as well as work on development of silicon models of single neurons [2]. These aVLSI systems are so successful because the mechanisms for signaling in the neural system, which are governed by Boltzmann statistics, can be captured by Metal Oxide Semiconductor Field Effect Transistor (MOSFET) circuits operating in their sub-threshold or low voltage regime. aVLSI is used in implementation of the silicon neurons and neural circuits because computations like addition, subtraction, expansion and compression are natural for analog circuits. It is these natural computational capabilities that make analog circuits so desirable for implementing biological functions. Since biological neurons and aVLSI circuits have similar data processing strategies, an analog circuit is a good representation of a biological system. The technology used for these ICs is Complimentary Metal Oxide Semiconductor (CMOS), a method of design that has many advantages for simulating biological functions [3]. Some of these advantages include: wide use in data processing applications, cost-effectiveness, well-understood fabrication processes, and low power consumption.

\section{BACKGROUND}

Our focus in this work is on the design and execution of a dendrite segment emulated in aVLSI. Much current work on dendrites is focused on investigating the active properties of the dendrites and their role in both computational processing and in learning $[4,5]$. This emphasis has benefited from new techniques in both data acquisition and imaging [6] and development of methods for modeling the dendritic functionality. Some current research topics include studies of the role of the active conductances and their relationship to computation; the functionality of various compartments in the dendrite; the relationship between timing and information coding [7] and; the role of antidromic or backpropagating spikes in plastic changes to the dendrite morphology. The use of neuromorphic engineering can enhance the ability to investigate, particularly, the methods of computational processing by developing analogs of the dendrite functionality, demonstrating the processes in both physical (aVLSI) and mathematical formulations, and testing outcomes using the aVLSI implementations.

\section{CIRCUIT COMPONENTS}

There are two fundamental types of circuits used to emulate the dentrite, active circuits that simulate the voltage dependent conductances associated with protein channels and passive circuits that simulate the transmission line aspects of the dendrite. An active circuit which includes $\mathrm{Na}$ and $\mathrm{K}$ channels [8] is shown in block diagram form in Fig. 1. It is constructed from transconductance amplifiers [3] in either the comparator (A) or delay (B) configuration with current mirror circuits (C) that represent the biological conductance channels. One version of the passive circuit is constructed from simple Resistor-Capacitor (RC) networks as shown in Fig. 2.

Each of these circuit types can be adjusted to produce a circuit response that matches the results of experimentally obtained biological data.

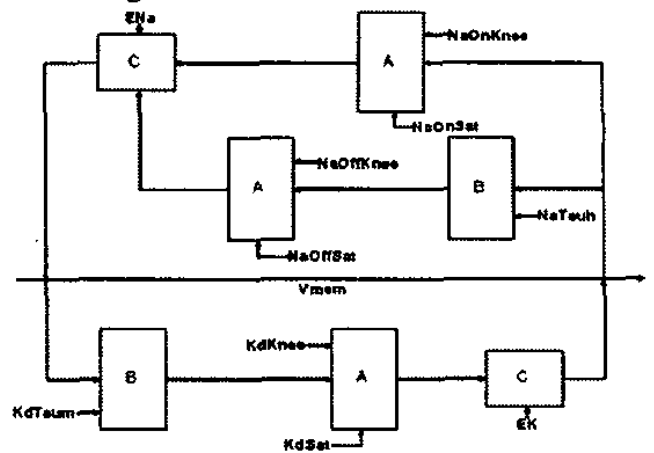

Fig. 1 Block diagram of an active circuit which emulates the voltage dependent $\mathrm{Na}$ (above $\mathrm{V}_{\text {meno }}$ ) and $\mathrm{K}$ (below $\mathrm{V}_{\text {mem }}$ ) channels [8].

\section{PARAMETER ADJUSTMENT}

By setting the individual parameters that are shown as inputs on the A, B and C segments in Fig. 1, bio-physical parameters of the dendrite segment can be adjusted to reproduce the desired output. In this section, we describe the technique for adjustment of the membrane resting potential, the threshold voltage for spiking, the width of a generated spike, the extent of hyperpolarization during the refractory period and passive signal attenuation.

1) Membrane Resting Potential

The resting potential, $V_{\text {rest }}$, can be adjusted by varying the physical size and static gate voltage of the MOSFETs which replicate the voltage dependent channels in the circuit. For example, in the block diagram of Fig. 1, an adjustment of $\mathrm{V}_{\text {rest }}$ occurs when the size of the conductance MOSFET in C block of the $\mathrm{K}$ circuit (portion below $\mathrm{V}_{\text {mem }}$ ) is changed. 


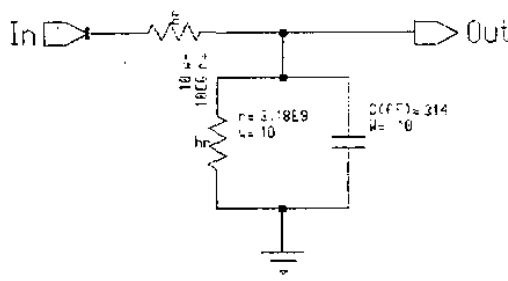

Fig 2. Diagram of passive circuit used to emulate the transmission line aspects of the dendrite.

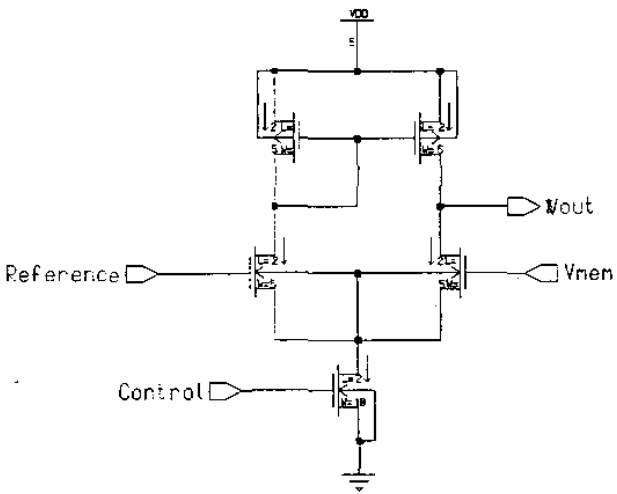

Fig. 3. Circuitry associated with the A blocks shown in Fig. 1. The reference input sets a switching point for the output for use in setting both on and off thresholds.

\section{2) Threshold Voltage}

A blocks in both the $\mathrm{Na}$ and $\mathrm{K}$ portions of Fig. 1 have reference values to indicate the voltage at which they will change state. The setting point $\mathrm{NaOnKnee}$ associated with the $\mathrm{A}$ block in the $\mathrm{Na}$ circuit (portion above $\mathrm{V}_{\text {mem }}$ ) sets the threshold voltage.

3) Spike Width

An adjustment to the delay time setting point (NaTauh) in the $\mathrm{B}$ block of the $\mathrm{Na}$ circuit (portion above $\mathrm{V}_{\text {mem }}$ in Fig. 1) will set the time that the spike voltage begins to fall toward $\mathrm{V}_{\text {rest. }}$ This allows the designer to have control over the spike width.

\section{4) Refractory Hyperpolarization Voltage}

The B block of the $\mathrm{K}$ circuit in Fig. 1 (portion below $V_{\text {mem }}$ ) controls the time that the $\mathrm{K}$ channel is allowed to remain open. If this channel remains open for a very long time, the membrane potential will fall to the $\mathrm{K}$ reversal potential. The value of KdTaum controls the time that the $K$ channel is open and thus the final value of $V_{\text {mem }}$ before it returns to $\mathrm{V}_{\text {rest. }}$.

5) Passive Attenuation

The passive attenuation of a signal as it propagates toward the soma can be set by adusting the resistance and capacitance of transmission line segments such as that illustrated in Fig. 2.

Fig. 5 shows output from the active circuit with two different settings for the threshold voltage and the resting potential.The figure caption describes the parameters for each output.

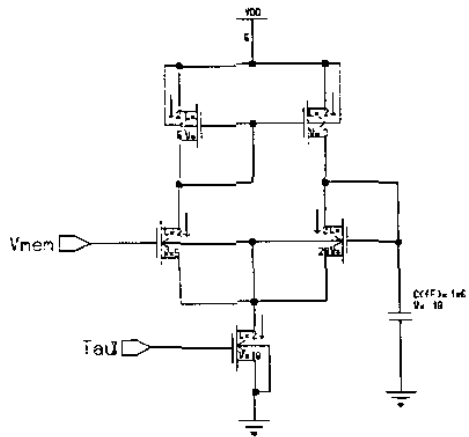

Fig. 4 Circuitry associated with the B blocks shown in Fig. 1. The Tau input sets a delay time for a signal passing through the circuit.

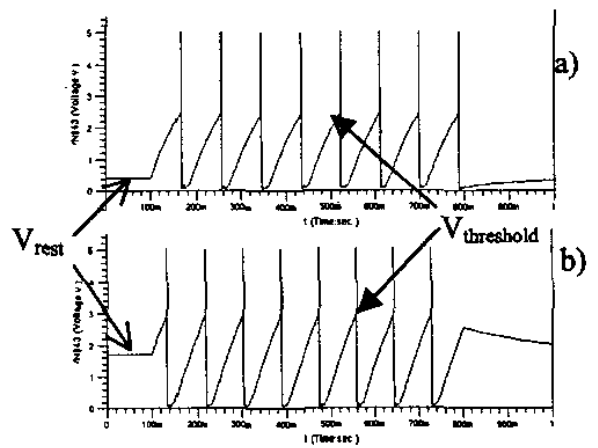

Fig. 5. Plots of $V_{\operatorname{men}}$ from the circuit of Fig. 1 with a constant current input and a) $\left.\mathrm{V}_{\text {reas }}=0.4 \mathrm{~V}, \mathrm{~V}_{\text {thentoud }}=2.5 \mathrm{~V}, \mathrm{~b}\right) \mathrm{V}_{\mathrm{res}}=1.7 \mathrm{~V}, \mathrm{~V}_{\text {trectold }}=3.0 \mathrm{~V}$.

\section{CONCLUSION}

Using passive and active circuits, the full functionality of a neuron can be demonstrated. These circuit components can be used modularly to construct segments of dendrites and full implementations of neurons. The applications extend from practical circuits for neural functions to the modeling of neural behavior for enhanced understanding of biological mechanisms. Full connectivity for a segment of dendrite will be demonstrated in the poster.

\section{REFERENCES}

[1] Mahowald, M. and Mead, C. A., "The Silicon Retina," Scientific American, May 1991, pp. 76-82.

[2] Mahowald, M. and Mead, C. A., "A Silicon Neuron," Nature, Vol. 354 , pp 515-518, 1991.

[3] Mead, C. A.. Analog VLSI and Neural Systems, Addison-Wesley, Reading, MA, 1989.

[4] Yuste, R and Tank, D. W., "Dendritic Integration in Mammalian Neurons, a Century after Cajal," Neuron, Vol. 16, pp 701-716, April, 1996. [5] Koch, C., Biophysics of Computation, Oxford University Press, New York and Oxford, 1999.

- [6] Mel, B. W., "Information Porcessing in Dentritic Trees," Neural Computation, Vol. 6, pp 1031-1085, 1994.

[7] Gabbiani, F., Krapp, H. G., Koch, C. and Laurent, G., "Multiplicative computation in a visual neuron sensitive to looming," Nature, Vol. 420, pp $320-324,2002$

[8] Shin, J, and Koch, C., "Dynamic Range and Sensitivity Adaptation in a Silicon Spiking Neuron," IEEE Trans. On Neural Networks, Vol. 10, No. 5 , pp 1232-1238, 1999. 